\title{
Information of Valence Charge of 3d Transition Metal Elements Observed in L- Emission Spectra
}

\author{
Masami Terauchi ${ }^{1 *}$ \\ 1. Institute of Multidisciplinary Research for Advanced Materials, Tohoku University, Sendai, Japan. \\ * Corresponding author: terauchi@m.tohoku.ac.jp
}

Bonding state and valence charge of $3 \mathrm{~d}$ transition metal (3d-TM) elements is a key for evaluating positive electrode materials for Li-ion battery, because a change of valence charge of 3d-TM elements is an origin of charge and discharge performance of the battery. Information of valence electrons of 3d-TM elements is included in L-emissions of the elements. There are four types of $\mathrm{L}$-emissions of $\mathrm{L} \alpha, \mathrm{L} \beta, \mathrm{L} \ell$ and $\mathrm{L} \eta$. Those are classified into two having close emission energies of $L \alpha, \beta$ and $L \ell, \eta$. L $\alpha, \beta$ emission due to $3 \mathrm{~d}_{5 / 2,3 / 2} \rightarrow 2 \mathrm{p}_{3 / 2,1 / 2}\left(\mathrm{~L}_{2,3}\right)$ electronic transition is a suitable probe of valence states (bonding states). The charge state of $3 \mathrm{~d}$ element is important not only for battery materials but also for discussing conductivity and magnetism of $3 \mathrm{~d}$ transition metal compounds. On the other hand, $L \ell, \eta$ emission due to transitions from a shallow inner-shell of $\mathrm{M}_{1}\left(3 \mathrm{~s}_{1 / 2}\right)$ to a deeper inner-shell of $\mathrm{L}_{2,3}$ can give different information from that of L $\alpha, \beta$ emissions [1]. Here, L-emission spectra of $3 \mathrm{~d}$ transition metal elements from Sc to $\mathrm{Zn}$ and some oxides were measured to examine a relation between L-emission intensities of $L \alpha, \beta \& L \ell, \eta$ and a number of $3 \mathrm{~d}$ electrons $\left(\mathrm{N}_{3 \mathrm{~d}}\right)$ of those elements by using a soft X-ray emission spectrometer attached to a scanning electron microscope [2,3]. For aiming a quantitative evaluation, spectral intensities were detected by a MCP detector in photon counting mode, which means that the integrated intensity of an emission peak corresponds to a number of emitted X-ray photons or related electronic transitions in a specimen examined [4].

Figure 1 shows L-emission spectra of simple metal materials of $\mathrm{Sc}\left(\mathrm{Z}=21,3 \mathrm{~d}^{1} 4 \mathrm{~s}^{2}\right), \mathrm{Ti}\left(\mathrm{Z}=22,3 \mathrm{~d}^{2} 4 \mathrm{~s}^{2}\right), \mathrm{V}$ $\left(Z=23,3 d^{3} 4 s^{2}\right), C r\left(Z=24,3 d^{5} 4 s^{1}\right)$, and $M n\left(Z=25,3 d^{5} 4 s^{2}\right)$. Each spectrum intensity is normalized by its maximum intensity in each spectrum. It is clearly seen that L-emission energy increases with an increase of $\mathrm{Z}$, which is caused by an increase of the binding energy of $\mathrm{L}_{2,3}$ level according to $\mathrm{Z}$. Furthermore, the intensity ratio of $L \alpha, \beta / L \ell, \eta$ increase according to an increase of $N_{3 d}$. This tendency of $L \alpha, \beta / L \ell, \eta$ is reasonable because the electron number contributed for L $\ell, \eta$ emission is constant of two $3 \mathrm{~s}$ electrons whereas that for $L \alpha, \beta$ emission, $\mathrm{N}_{3 \mathrm{~d}}$, increases with $\mathrm{Z}$. Thus, the intensity ratio of $\mathrm{L} \alpha, \beta / \mathrm{L} \ell, \eta$ can be an indicator for evaluating $\mathrm{N}_{3 \mathrm{~d}}$. However, the increase of $\mathrm{L} \alpha, \beta / \mathrm{L} \ell, \eta$ according to $\mathrm{N}_{3 \mathrm{~d}}$ was not linear. As two transition of $3 \mathrm{~d}_{5 / 2,3 / 2} \rightarrow \mathrm{L}_{2,3}$ and $\mathrm{M}_{1}\left(3 \mathrm{~s}_{1 / 2}\right) \rightarrow \mathrm{L}_{2,3}$ are competing in L-emission process, the transition probability of $3 \mathrm{~d}_{5 / 2,3 / 2} \rightarrow \mathrm{L}_{2,3}$ should change according to a change of $\mathrm{N}_{3 \mathrm{~d}}$. Then, intensity of L $\alpha, \beta$ compared with the total $L$-emission intensity of $L \alpha, \beta+L \ell, \eta$, which should reflect not only the number of $3 \mathrm{~d}$ electrons but also a change of transition probability due to a change of $\mathrm{N}_{3 \mathrm{~d}}$, was evaluated. As shown in Fig.2, the values of $L \alpha, \beta /(L \alpha, \beta+L \ell, \eta)$ increase almost linearly according to $N_{3 d}$ from $\mathrm{Sc}$ to $\mathrm{Ni}$, except $\mathrm{Cr}$, The deviation of $\mathrm{Cr}$ value from an expected gradual increase may reflect a characteristic electron configuration in $3 d$ orbitals. The linear tendency of $L \alpha, \beta /(L \alpha, \beta+L \ell, \eta)$ was fitted by a linear equation and applied to some oxides. In case of $3 \mathrm{~d}$ metal oxides, the evaluation by using the equation was over estimate the number of $3 \mathrm{~d}$ electrons. It can be assigned to be due to a charge transfer from ligand oxygen atoms to a $3 \mathrm{~d}$ transition metal element owing to a core-hole effect in the intermediate state as reported by Grebennikov et al. [5,6]. 
References:

[1] M Terauchi et al., IOP Conf. Series: Materials Science and Engineering 304 (2017), p. 012018.

[2] M Terauchi et al., Microscopy and Microanalysis 20 (2014), p. 692.

[3] M Terauchi et al., Microscopy and Microanalysis 22(S3) (2016), p. 414.

[4] M Terauchi, Microscopy, submitted.

[5] V I Grebennikov et al., PHYSICS OF THE SOLID STATE 45 (2003), p. 1048.

[6] This work was partly supported by the Research Program of "Dynamic Alliance for Open Innovation Bridging Human, Environment and Materials" in "Network Joint Research Centre for Materials and Devices".

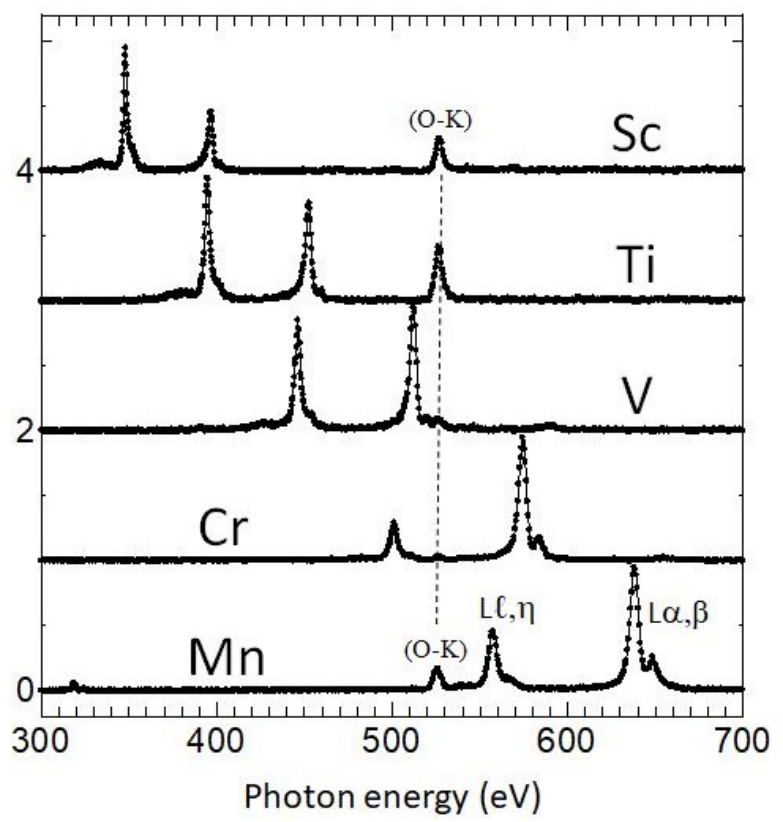

Figure 1. L-emission spectra of simple metal specimens of $\mathrm{Sc}\left(\mathrm{Z}=21,3 \mathrm{~d}^{1} 4 \mathrm{~s}^{2}\right)$, Ti $\left(\mathrm{Z}=22,3 \mathrm{~d}^{2} 4 \mathrm{~s}^{2}\right), \mathrm{V}$ $\left(Z=23,3 d^{3} 4 s^{2}\right), C r\left(Z=24,3 d^{5} 4 s^{1}\right)$, and $M n\left(Z=25,3 d^{5} 4 s^{2}\right)$. Each spectrum intensity is normalized by its maximum intensity in each spectrum.

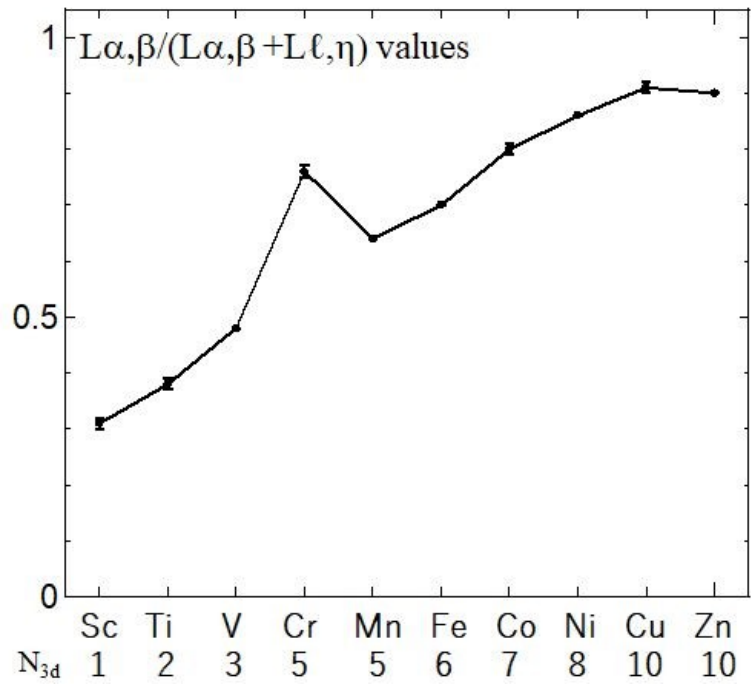

Figure 2. Experimentally obtained $L \alpha, \beta /(L \alpha, \beta+L \ell, \eta)$ values for $3 d$ transition metal materials. 